\title{
Use of the painDETECT tool in rheumatoid arthritis suggests neuropathic and sensitization components in pain reporting
}

This article was published in the following Dove Press journal: Journal of Pain Research

14 October 2014

Number of times this article has been viewed

\section{Saqa Ahmed* \\ Tejal Magan* \\ Mario Vargas \\ Abiola Harrison \\ Nidhi Sofat}

Infection and Immunity Research Institute, St George's, University of London, London, UK

*These authors contributed equally to this work

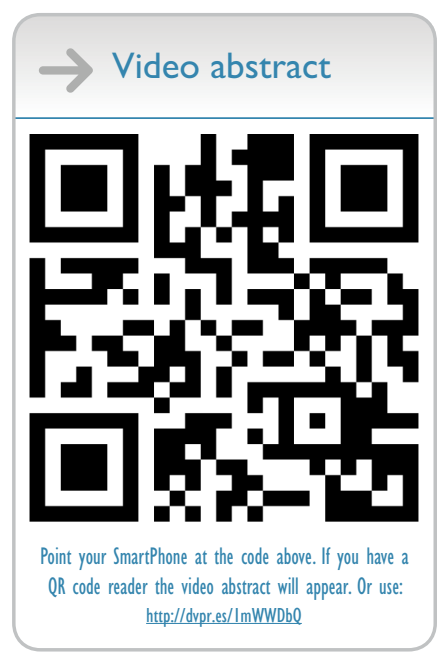

Correspondence: Nidhi Sofat Infection and Immunity Research Institute, St George's, University of London, Cranmer Terrace, London SWI7 ORE, UK Email nsofat@sgul.ac.uk
Abstract: Rheumatoid arthritis (RA) is an inflammatory autoimmune condition typified by systemic inflammation targeted toward synovial joints. Inhibition of proinflammatory networks by disease-modifying antirheumatic drugs, eg, methotrexate and biologic therapies, including tumor necrosis factor- $\alpha$ inhibitors, often leads to suppression of disease activity observed at the clinical level. However, despite the era of widespread use of disease-modifying treatments, there remain significant groups of patients who continue to experience pain. Our study formulated a pain assessment tool in the arthritis clinic to assess feasibility of measurements including the visual analog scale (VAS) and painDETECT to assess multimodal features of pain in people with established RA $(n=100)$. Clinical measures of disease activity (Disease Activity Score in 28 Joints [DAS28]) were also recorded. Our data showed that despite the majority of subjects on at least one disease-modifying agent, the majority of patients reported severe pain (54\%) by VAS, despite well-controlled clinical disease, with mean DAS28 2.07 \pm 0.9 . Using the painDETECT questionnaire, $67 \%$ of patients had unlikely neuropathic pain. A significant proportion of subjects (28\%) had possible neuropathic pain and 5\% had features of likely neuropathic pain by painDETECT scoring. We found a positive correlation between VAS and painDETECT $\left(R^{2}=0.757\right)$. Of note, the group who had likely or probable neuropathic pain also showed significantly increased pain reporting by VAS $(P<0.01)$. Subjects who were clinically obese (body mass index $>30$ ) also had statistically higher proportions of pain reporting (VAS $89.0 \pm 0.7 \mathrm{~mm}$ ) compared with subjects who had a normal body mass index (VAS $45.2 \pm 21.8 \mathrm{~mm}$ ), $P<0.05$. Our findings suggest that multimodal features of pain perception exist in RA, including neuropathic and sensitization elements, perhaps explaining why a subgroup of people with RA continue to experience ongoing pain, despite their apparent suppression of inflammation.

Keywords: rheumatoid arthritis, pain, sensitization, painDETECT, neuropathic pain

\section{Introduction}

Rheumatoid arthritis (RA) is an archetypal autoimmune-mediated proinflammatory condition. Clinically, it is typified by swelling, pain, and reduced function in affected joints. Uncontrolled RA causes disability and reduces quality of life, placing a high disease burden on affected populations. ${ }^{1}$ Disease-modifying antirheumatic drugs (DMARDs) and biologic drugs, including tumour necrosis factor inhibitors, can reduce disease activity and improve disability. However, despite a host of new immune-mediated therapies available to treat RA, significant numbers of patients exist who continue to experience pain, despite the use of DMARDs. ${ }^{2}$ The UK-based National Institute for Health and Care Excellence (NICE) guidance has outlined best practice, ${ }^{1}$ and several international guidelines for RA care exist to guide treatment. ${ }^{3}$ 
Current interventions achieve remission in $30 \%$ of patients but leave many, ie, $50 \%-60 \%$, with ongoing disease activity in the UK alone. ${ }^{4}$

An increasing challenge in RA management is to optimize disease remission and treatment of pain in a significant number of patients who report ongoing pain despite treatment with often expensive disease-modifying drugs. In a recent UK-based study of 1,189 people with RA, after 1 year of treatment with disease-modifying drugs, the level of pain reporting remained high. ${ }^{5}$ McWilliams et $\mathrm{al}^{5}$ showed that there was no significant change in reported pain levels despite the use of disease-modifying drugs. Such observations, now also from other groups, ${ }^{6,7}$ have led to the formulation of the hypothesis that people with RA have a heightened pain experience very early on in their disease. It is possible that people with early RA may have multiple components of pain, including neuropathic and sensitization elements. By sensitization we mean a process of heightened pain perception derived from hypersensitivity to stimuli by sustained activation of peripheral nociceptors, eg, in the arthritic joint. ${ }^{8,9}$ Merskey ${ }^{8}$ defined pain as "an emotional experience with an unpleasant sensation that is accompanied by an actual or potential damage or injury to tissue." It is fundamentally one of the most impairing symptoms among people with RA. Pain is a persisting symptom in people with RA, and up to $\sim 70 \%$ would like to see improvements in pain compared with other symptoms of RA. ${ }^{2,10,11}$

In this article, we propose methods by which pain assessment in the clinic can assist to establish the nature of pain phenotypes in RA. A number of groups have recently reported the use of the painDETECT questionnaire ${ }^{12}$ as a quantitative tool for measuring noninflammatory, neuropathic, or sensitization elements of pain. The painDETECT questionnaire has already been investigated in distinct groups of people with musculoskeletal pain, including fibromyalgia, ${ }^{13}$ back pain, ${ }^{14}$ and osteoarthritis. ${ }^{15}$ All of the studies described have reported neuropathic/sensitization features of pain in the musculoskeletal conditions described, including in people who were already being treated with analgesic drugs. However, to our knowledge, no reports on the use of the painDETECT questionnaire in pain reporting in RA have been published to date. We used the published version of the painDETECT questionnaire, which was developed by Freynhagen et al, ${ }^{12}$ and used it for the first time in people with RA to assess pain characteristics in this autoimmune condition. Our work has found that RA pain is likely to be a multimodal entity with features of inflammation, neuropathic pain, and sensitization. We propose that wider use of painDETECT in the clinical setting of arthritis clinics may assist in identifying neuropathic or sensitization pain features in people with RA to help optimize their future pain management.

\section{Methods}

We conducted a study of 100 participants (32 males and 68 females) with confirmed RA based on American College of Rheumatology/European League Against Rheumatism criteria $^{3}$ from a tertiary care rheumatology center at St George's Hospital, London, UK. A patient reporting tool was formulated, consisting of 24 questions, and was used to evaluate the effectiveness of pain management and monitoring. Pain analysis tools, including the visual analog scale (VAS) $(0-100 \mathrm{~mm})$ and the painDETECT questionnaire, were included to evaluate pain intensity, duration, and nature in the group. Additional demographic data and Disease Activity Score in 28 Joints (DAS28) for RA duration, use of DMARDs, and demographic data were also collected. The data collection tools used in this study are summarized in Table 1. Full ethical approval for this study was provided by the London Surrey Borders Ethics Committee (REC No 115212). Participants were interviewed during their routine clinic appointment and identified from arthritis clinic records. They were eligible to participate in the study if their treatment had been stable for the previous 3 months and they did not require corticosteroid therapy.

The VAS is a widely used tool in pain assessment for RA and forms part of the pain reporting tool in DAS28. ${ }^{16,17}$ To date, additional tools such as the painDETECT questionnaire, which is now increasingly used to assess neuropathic elements of pain, have not been reported widely in assessments of people with RA. To investigate the value of pain assessment tools in addition to VAS, we explored the use of the painDETECT questionnaire in a group of people with established RA. We set parameters for VAS and painDETECT based on published reporting for subsets of pain. Previous studies have described

Table I Stratification of patients based on pain questionnaires and disease activity indices

\begin{tabular}{lll}
\hline Scoring system & Range & Interpretation \\
\hline Visual analog scale & $0-30$ & Mild pain \\
& $31-53$ & Moderate pain \\
& $54-100$ & Severe pain \\
painDETECT & $\leq 12$ & Unlikely neuropathic pain \\
& $13-18$ & Possible neuropathic pain \\
& $\geq 19$ & Likely neuropathic pain \\
Disease Activity Score & $\leq 3.2$ & Low disease activity \\
in 28 Joints & $3.3-5.1$ & Moderate disease activity \\
& $>5.1$ & High disease activity \\
\hline
\end{tabular}


VAS measurements for pain as mild $(0-30 \mathrm{~mm})$, moderate (31-53 mm), and severe $(54-100 \mathrm{~mm}) .{ }^{18}$ With respect to painDETECT, scores suggesting likely neuropathic pain are $\geq 19$, probable neuropathic pain 13-18, and unlikely neuropathic pain $\leq 12$. The painDETECT questionnaire was validated and developed in Germany. ${ }^{12}$ It is a patient-based questionnaire that could be used by specialists as well as nonspecialists. It consists of nine items of which seven are weighted sensory descriptor items and two items relate to the spatial (radiating) and temporal characteristics of the individual pain pattern. From the answers, an overall score is generated ranging from 1 to 38. According to the researchers who developed the painDETECT questionnaire, ${ }^{12}$ a score of $\leq 12$ indicates that a neuropathic component is unlikely (with a true negative sensitivity of $<15 \%$ ), a score of $\geq 19$ suggests that the pain is likely to have a neuropathic component (with a true positive sensitivity of $>90 \%$ ), and a score of 13-18 indicates that there is a slight chance that there is a neuropathic component. This tool for assessing neuropathic pain when compared with clinical diagnosis has $85 \%$ sensitivity and $80 \%$ specificity. ${ }^{12}$

Pain reporting data were also compared with recorded DAS28 scores in our patient group. The simplified DAS28 is a modified derivative of the original DAS, assessing 28 designated joints from the 44 joints in original reports. ${ }^{19,20}$ This tool is generally used by the physician or specialist nurse to assess the number of swollen and tender joints out of the 28 . It also takes account of the erythrocyte sedimentation rate (ESR) or the C-reactive protein (CRP). The ESR and CRP can both be used to measure the degree of inflammation in the serum, with usually one of the readings, either ESR or CRP, being used to calculate the DAS28 for the patient according to the formula given here. The patient's global assessment of health on a $0-100$ scale is also measured. In addition, the VAS component of the DAS28 is usually the only pain assessment tool used in many clinical settings for RA care. Once the scores are calculated, they are then used in a mathematical formula to produce the overall disease activity score:

$$
\begin{aligned}
\mathrm{DAS}= & 0.56 \times \sqrt{\mathrm{TJC}}+0.28 \times \sqrt{\mathrm{SJC}}+0.70 \times \ln (\mathrm{ESR}) \\
& +0.014 \times \mathrm{GH}
\end{aligned}
$$

where $\mathrm{TJC}=$ tender joint count, $\mathrm{SJC}=$ swollen joint count, $\mathrm{ESR}=$ erythrocyte sedimentation rate, and $\mathrm{GH}=$ general health.

Values of DAS28 $>5.1$ indicate high disease activity, 3.2-5.1 means moderate disease activity, 2.6-3.2 means low disease activity, and a score of $<2.6$ means the patient is in remission. ${ }^{21}$

\section{Statistical analysis}

All data were analyzed using GraphPad Prism 7 software. Continuous data were described and analyzed using parametric statistics. Variables following a normal distribution were assessed as mean \pm standard deviation. When comparing three or more groups, one-way analysis of variance was used. Nonparametric data were presented as descriptive statistics (median, interquartile range) and analyzed using the chi-square test. The categorical variables were recorded as numbers (n) and percentages (\%) and were compared using the chi-square test if they had three or more categories. Statistical significance was considered at $P$-values $<0.05$.

\section{Results}

The results from our study demonstrate that participants with RA reported relatively high pain levels, despite the widespread use of disease-modifying drugs in this group of patients (Tables 2 and 3). The majority of participants with RA, namely 54\%, reported "severe pain" on the VAS, which identifies people with a VAS of 54-100 $\mathrm{mm}$ as having the highest severity of pain. The mean DAS28 in the group was 2.07 \pm 0.9 . The majority of subjects had a duration of diagnosis $\geq 5$ years (84\%). All participants evaluated had been stable on DMARD therapy for at least 3 months prior to completing the study and had not required a change in their treatment or addition of corticosteroid therapy during that time. The majority of participants were being treated with disease-modifying drugs, including the commonest agent, methotrexate (82\%). Table 2 shows a summary of the DMARD agents being taken by the group as a whole, with the majority being on methotrexate and combination therapy in the form of additional oral DMARDs, eg, methotrexate, sulfasalazine, hydroxychloroquine, penicillamine, or ciclosporin. Additionally, all the subjects who were on biologic drugs and therefore by definition had more severe disease reported pain in the "severe pain" group. That is, of 39 participants who were taking one of the biologic agents infliximab, abatacept, or tocilizumab, $76.9 \%$ reported "severe pain". Our data suggest that initial treatment may not have been efficient in achieving disease control measured by DAS28 score, leading to the decision to use combination DMARD therapy. The use of pain-relieving medications was also greatest in the "severe pain" group, which had the highest use of analgesic medications including paracetamol, ibuprofen, and other pain-relieving agents. The painDETECT questionnaire was used to evaluate for a possible neuropathic element of pain among the RA group. 
Table 2 Demographic characteristics for participants with rheumatoid arthritis and their use of disease-modifying drugs ${ }^{\mathrm{a}}$

\begin{tabular}{|c|c|c|c|c|c|}
\hline & \multirow{2}{*}{$\begin{array}{l}\text { All } \\
(N=\mid 00)\end{array}$} & \multicolumn{3}{|l|}{ VAS } & \multirow[t]{2}{*}{$P$-value } \\
\hline & & $\begin{array}{l}\text { Mild pain } \\
(\mathrm{N}=19)\end{array}$ & $\begin{array}{l}\text { Moderate pain } \\
(\mathrm{N}=27)\end{array}$ & $\begin{array}{l}\text { Severe pain } \\
(N=54)\end{array}$ & \\
\hline DMARDs & & & & & $0.093 \chi^{2}$ \\
\hline Methotrexate & 82 & 10 & 22 & 50 & \\
\hline Sulfasalazine & 31 & 9 & 8 & 14 & \\
\hline Hydroxychloroquine & 38 & 8 & 15 & 15 & \\
\hline Penicillamine & 8 & 0 & 2 & 6 & \\
\hline Ciclosporin & 13 & 0 & 3 & 10 & \\
\hline Biologics & & & & & $0.043 \chi^{2}$ \\
\hline Infliximab & 20 & 0 & 1 & 19 & \\
\hline Abatacept & 17 & I & 7 & 9 & \\
\hline Tocilizumab & 2 & 0 & 0 & 2 & \\
\hline Pain medication & & & & & $0.393 \chi^{2}$ \\
\hline Paracetamol & 79 & 14 & 20 & 45 & \\
\hline Ibuprofen & 26 & 4 & 9 & 13 & \\
\hline Other & 48 & 9 & 7 & 32 & \\
\hline painDETECT & $8.77 \pm 5.58$ & $2.84 \pm 1.30$ & $5.00 \pm 1.96$ & $12.74 \pm 4.46$ & $<0.000$ I $F$ \\
\hline DAS28 & $2.09 \pm 0.96$ & $1.95 \pm 1.08$ & $2.16 \pm 0.86$ & $2.16 \pm 0.86$ & \\
\hline
\end{tabular}

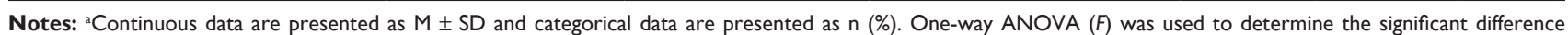
between the three groups (mild, moderate, and severe pain) for the parametric data. Categorical data were analysed using the chi-square test $\left(\chi^{2}\right)$. painDETECT one-way ANOVA: $(F[2,97]=349.6, P<0.0001)$. The post hoc comparison for painDETECT using the Tukey test indicated that the significant difference is between all the conditions: mild vs moderate $(M=-32.83, S D=1.947)$, mild vs severe $(M=-60.54, S D=2.312)$, and moderate vs severe $(M=-27.71, S D=2.099)$.

Abbreviations: VAS, visual analog scale; DMARDs, disease-modifying antirheumatic drugs; DAS28, Disease Activity Score in 28 Joints; M, mean; SD, standard deviation; ANOVA, analysis of variance.

Figure 1 shows that $33 \%$ of participants reported possible or likely neuropathic pain by painDETECT. Additionally, there was a clear positive trend between VAS scores and painDETECT scores, suggesting that the high level of pain reported on the VAS also correlated with high neuropathic pain scores $\left(R^{2}=0.757\right)$. One of the components of the painDETECT questionnaire is the body map, where subjects can mark body areas in which they felt pain. The majority of participants with probable neuropathic pain or likely neuropathic pain reported pain around their knees, ankles,

Table 3 Characteristics for age, body mass index, sex, smoking history, and duration of diagnosis for rheumatoid arthritis study group ${ }^{\mathrm{a}}$

\begin{tabular}{|c|c|c|c|c|c|}
\hline & \multirow[t]{2}{*}{ All $(N=100)$} & \multicolumn{3}{|l|}{ VAS } & \multirow[t]{2}{*}{$P$-value } \\
\hline & & $\begin{array}{l}\text { Mild pain } \\
(\mathrm{N}=19)\end{array}$ & $\begin{array}{l}\text { Moderate pain } \\
(\mathrm{N}=27)\end{array}$ & $\begin{array}{l}\text { Severe pain } \\
(N=54)\end{array}$ & \\
\hline Age, years & & & & & $0.358 \chi^{2}$ \\
\hline$\leq 30$ & 7 & I & 3 & 3 & \\
\hline $31-40$ & 15 & 5 & 4 & 6 & \\
\hline $4 I-50$ & 52 & 12 & 12 & 28 & \\
\hline $51-60$ & 20 & 1 & 7 & 12 & \\
\hline $6 I-70$ & 6 & 0 & 1 & 5 & \\
\hline BMI & $24.3 I \pm 2.3 I$ & $23.45 \pm 1.31$ & $23.78 \pm 1.68$ & $24.88 \pm 2.69$ & $0.0231 F$ \\
\hline Sex & & & & & $0.833 \chi^{2}$ \\
\hline Male & 32 & 7 & 9 & 16 & \\
\hline Female & 68 & 12 & 18 & 38 & \\
\hline \multicolumn{6}{|l|}{ Smoking history } \\
\hline Yes & 10 & $\mathrm{I}$ & 1 & 8 & $0.253 \chi^{2}$ \\
\hline No & 90 & 18 & 26 & 46 & $0.858 \chi^{2}$ \\
\hline Duration of RA & & & & & $0.977 \chi^{2}$ \\
\hline 2-4 years & 16 & 3 & 4 & 9 & \\
\hline$\geq 5$ years & 84 & 16 & 23 & 45 & \\
\hline
\end{tabular}

Notes: ${ }^{a}$ Continuous data are presented as $M \pm S D$ and categorical data are presented as $\mathrm{n}(\%)$. One-way ANOVA was used to determine the significant difference between the three groups (mild, moderate, and severe pain) for the parametric data. Categorical data were analyzed using the chi-square test $\left(\chi^{2}\right)$. BMI one-way ANOVA: $(F[2,97]=3.919$, $P=0.023 \mathrm{I})$. The post hoc comparison for the BMI using the Tukey test indicated that the statistical difference is between the mild and the severe $(M=-I .432$, SD $=0.598 \mathrm{I})$. However, there was no statistical difference between mild vs moderate $(M=-0.3267, S D=0.67 I 5)$ and between moderate vs severe $(M=-1$. 106 , $S D=0.5285)$.

Abbreviations: VAS, visual analog scale; BMI, body mass index; RA, rheumatoid arthritis; M, mean; SD, standard deviation; ANOVA, analysis of variance. 

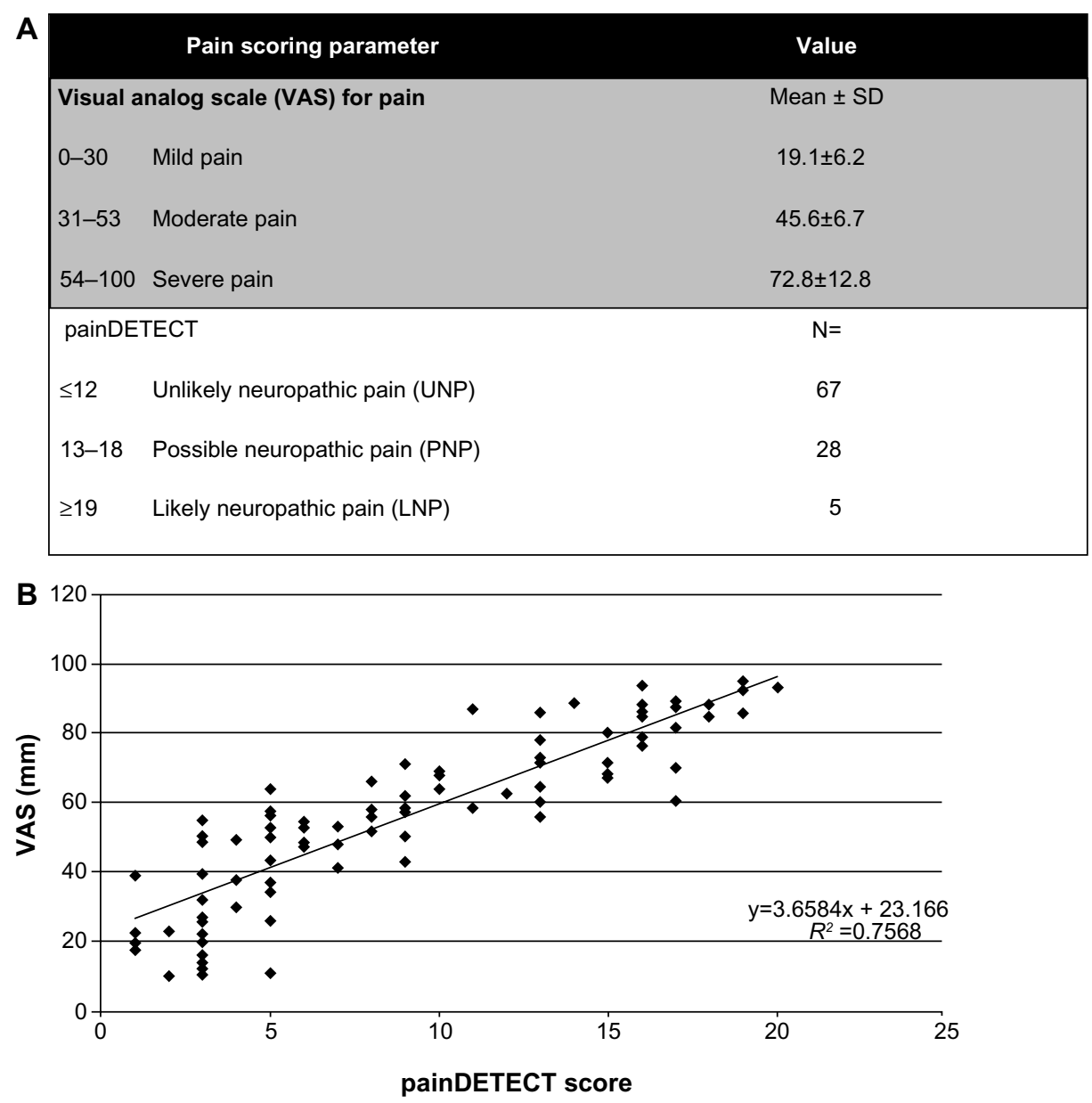

Figure I (A) Table showing categorization of rheumatoid arthritis group by pain stratification using visual analog scale (VAS) and painDETECT questionnaires. The numbers (N) represent number of participants in the study with the scoring for unlikely neuropathic pain (UNP), possible neuropathic pain (PNP), and likely neuropathic pain (LNP), respectively. (B) Scatter graph showing relationship between VAS $(\mathrm{mm})$ and painDETECT for the rheumatoid arthritis group. The $R^{2}$ value shows a positive correlation between VAS and painDETECT.

thighs, lower back, wrists, and shoulders on body mapping, suggesting widespread pain in keeping with likely sensitization in our RA group.

Analysis of sex differences in pain reporting did not identify any significant differences between men and women (Figure 2). When asked about their current pain compared with worst ever pain in their joints, a significant number of participants reported an improvement in their pain reporting at current levels (Figure 2B), suggesting that the majority of subjects had experienced higher pain levels previously. We also assessed the group for evidence of obesity by body mass index (BMI), since higher levels of obesity are known to correlate with higher pain reporting. ${ }^{22}$ We found that the reporting of pain by VAS increased according to subgroupings for subjects who were overweight and obese (Figure 3). The differences between the "normal" BMI group and the overweight or obese BMI group were statistically significant $(P<0.05)$.

When participants were grouped according to those on oral DMARDs alone (ie, methotrexate, sulfasalazine, hydroxychloroquine, penicillamine), biologic drugs, and DMARDs (ie, methotrexate and infliximab or tocilizumab or abatacept), the mean VAS for the oral DMARD group was $52.0 \pm 21.3 \mathrm{~mm}$ (Figure 4A). For the biologic and oral DMARD group, the mean VAS was $68.2 \pm 18.5 \mathrm{~mm}$, suggesting that participants on combination oral and biologic DMARD therapies had the highest VAS pain scores reported compared with participants not on any DMARD therapy ( $P \leq 0.0001)$. In comparison, $14 \%$ of the total group were not on any DMARD therapy, with a mean VAS of $30.4 \pm 19.5 \mathrm{~mm}$. The results of the painDETECT questionnaire divided subjects into three groups: unlikely neuropathic pain, possible neuropathic pain, and likely neuropathic pain. We found that the likely neuropathic pain group also reported the highest pain levels on VAS, with a mean VAS of $90.1 \pm 4.3 \mathrm{~mm}$ (Figure 4B), which was statistically significant compared with the unlikely neuropathic pain group $(P \leq 0.01)$. Interestingly, participants in the possible neuropathic pain group also demonstrated high VAS reporting, 
A

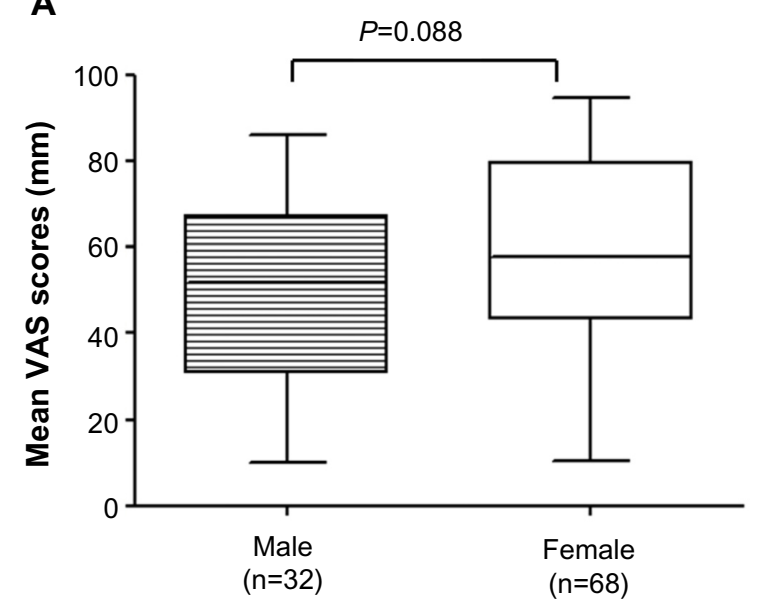

B

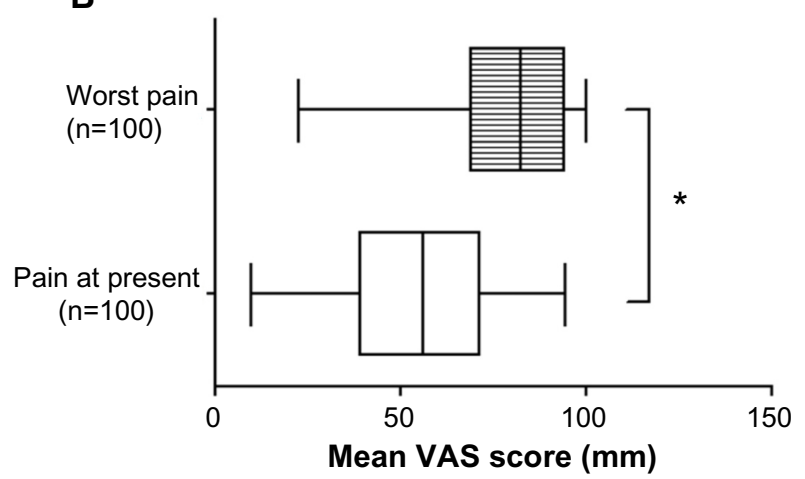

Figure 2 (A) Box plot graph of the distribution of the mean visual analog scale (VAS) according to sex. (B) Box and whiskers plot of the distribution of the mean VAS with respect to present pain at the time of questioning compared with worst pain experienced $(* P<0.05)$.

with a mean VAS of $77.8 \pm 10.3 \mathrm{~mm}$, which was statistically significant compared with the unlikely neuropathic pain group ( $P \leq 0.0001)$. Our results suggest that people with RA reporting high pain levels by VAS in the clinic could have neuropathic elements to their pain measured by tools such as painDETECT.

\section{Discussion}

We have found that high levels of pain reporting exist in RA in people with fairly well-controlled disease activity measured by DAS28. The "disconnect" observed between pain reporting and disease activity shows that specific components of pain in RA exist that may not be fully captured by the VAS. Our data from the painDETECT score showed that a large proportion of subjects with RA demonstrated likely or probable neuropathic pain features. Neuropathic pain classically refers to pain that is arising from the central nervous system, and it could be, as demonstrated by our study, that people with RA are sensitized to pain very early on in their disease. We have shown that despite the use of disease-modifying agents for RA, including both oral and biologic DMARDs, according to NICE guidelines, pain reporting remains high.

Our data suggest that there may be a noninflammatory or neuropathic component that is mediating certain components of pain perception in RA. In the group we studied, most subjects were on oral DMARD therapy, with the majority on at least methotrexate (82\%) plus additional diseasemodifying drugs. Of note, although the majority of subjects were prescribed oral DMARDs, 54\% reported high pain levels despite use of oral and biologic therapies. The use of analgesic medications in the moderate and severe pain groups was the highest.

Clinically, RA is identified with synovitis, which classically corresponds with inflammation-driven pain. Studies have shown that inflammation of the synovium leads to an acceleration of prostaglandin and bradykinin production, which leads to the activation of thin unmyelinated sensory nerves ( $\mathrm{C}$ fibers) in the synovium. ${ }^{23}$ Inflammation is pivotal in the overall perception of pain in RA. However, several studies, including the data herein, suggest that despite sufficient suppression of inflammation, there is still persistent pain even with the treatment of anti-inflammatory drugs, indicating that other factors mediating pain perception are involved in RA. ${ }^{24}$ It has been proposed that after a period of time, arthritic joints expand their total receptive field to the surrounding area of normal noninflamed tissue. The enhanced response to stimulation of joints that are inflamed could be mediated by peripheral sensitization. ${ }^{25}$ Additional increased pain responses to noninflamed tissue could be generated in the spinal cord, leading to central sensitization. Central sensitization is when normal inputs begin to produce aberrant feedback due to the excitation of the neurons in the central nervous system. ${ }^{26}$ Recent work in RA has shown that features such as anxiety, low mood, and depression have an impact on clinical pain reporting in RA and can influence brain activation in frontal regions, measured by brain functional neuroimaging studies. ${ }^{27}$ More recently, Rech et $\mathrm{al}^{28}$ showed that people with RA treated with biologic therapies could have early improvements in brain pain sensitization, observed by brain functional imaging using blood oxygen level-dependent signals, within days of treatment with biologic agents such as certolizumab pergol, well before an improved clinical response in the joints was observed.

From our results, we have identified that people with RA prescribed a combination of biologics and DMARDs had an overall high mean VAS score compared with the other groups, 
A

\begin{tabular}{|cc|}
\hline \multicolumn{2}{|c|}{ Body mass index - BMI score } \\
\hline Subscale & Mean (SD) \\
\hline Normal (18.5-24.9) & $23.1 \pm 1.8$ \\
\hline Overweight (25.0-29.9) & $26.2 \pm 1.1$ \\
\hline Obese ( $\geq 30)$ & $30.2 \pm 0.3$ \\
\hline The data are presented as mean (SD). Total sample size: 100 participants
\end{tabular}

B
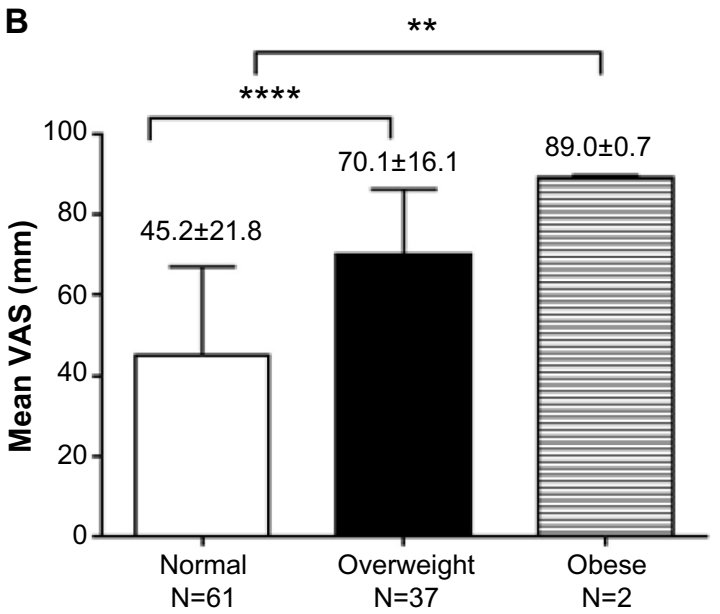

$\square$ Normal (18.5-24.9)

- Overweight (25.0-29.9)

$\boxminus$ Obese $(\geq 30)$

Figure 3 (A) Table showing categorization of rheumatoid arthritis group by body mass index (BMI). (B) Bar graph showing the distribution of visual analog scale (VAS) by $\mathrm{BMI}$ in the rheumatoid arthritis group. Participants were subgrouped by BMI into normal, overweight, and obese. The number ( $\mathrm{N}$ ) of participants within each BMI category is given. The mean (standard deviation [SD]) VAS in the groupings described is shown. There were statistically significant differences observed in the overweight and obese groups compared with controls $(* * P<0.01$, $* * * * P<0.000 \mathrm{I})$.

despite DAS28 scores suggesting good control of disease activity. This finding further suggests that there may be additional factors involved in RA pain processing other than inflammation alone. Biologics are powerful and expensive treatments usually given to patients with high DAS28 $(>5.1)$. A reduction in DAS28 of 1.2 points or more is considered as guidance for response criteria to biologic therapies and also considered as a benchmark for continuing treatment. ${ }^{29}$ DMARDs are a group of drugs that are unrelated and used in the treatment of RA to slow down the disease progression, limiting joint damage and improving function. ${ }^{30} \mathrm{~A}$ window of opportunity has emerged in RA treatment where earlier treatment with DMARDs equates with improved long-term outcomes. ${ }^{31,32}$ DMARDs help primarily to reduce inflammation and likely inflammatory pain. Together with agents such as nonsteroidal anti-inflammatory drugs, they help to limit and control the symptoms of RA. Additional analgesic agents that were reported to be of high usage in our study included paracetamol and codeine (can also be given in combination, known as co-codamol). ${ }^{33}$ Apart from central and peripheral sensitization, pain in RA can be further exacerbated by comorbidities, including obesity, fibromyalgia, diabetes mellitus, smoking, and secondary osteoarthritis. ${ }^{5}$ Psychological and physiological factors also contribute to the perception of pain, which is seen in patients with RA who also suffer from depression and anxiety. ${ }^{34}$ Clinicians could evaluate people with RA using painDETECT in addition to current tools being used to assess disease activity in the future. If they showed evidence of likely neuropathic pain, it could be addressed potentially with a trial of analgesics targeted at neuropathic pain. Interestingly, of the participants in our study who had severe pain, approximately $30 \%$ had been prescribed amitriptyline.

Potential limitations of our study are that numbers of patients were relatively small with 100 participants recruited. However, this was a feasibility study to test the practicality of using the VAS and painDETECT in a clinical setting where many parameters are already being used to 

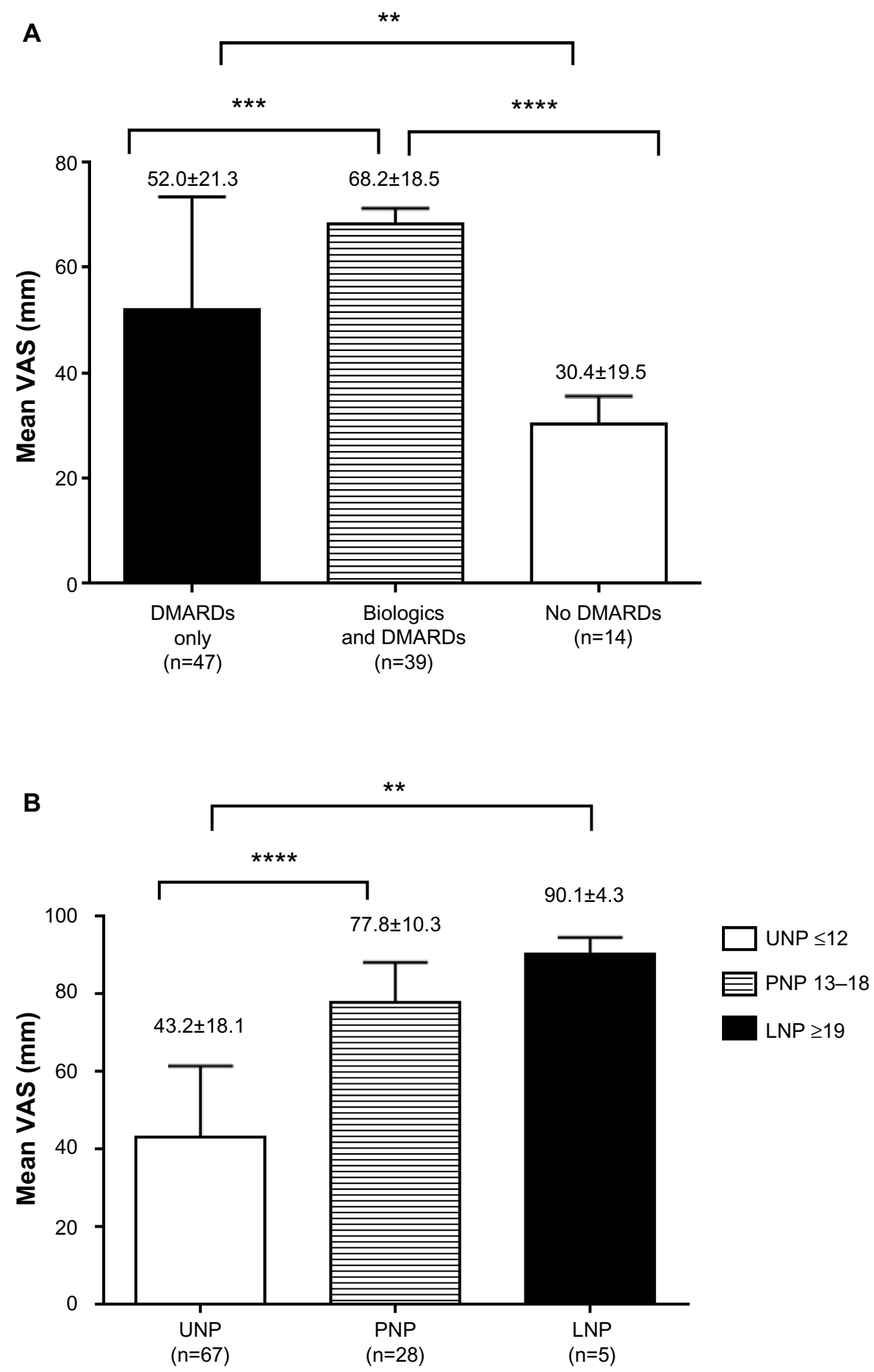

Figure 4 (A) Bar graph showing mean \pm standard deviation pain scores for visual analog scale (VAS) and their relationship to use of disease-modifying antirheumatic drug

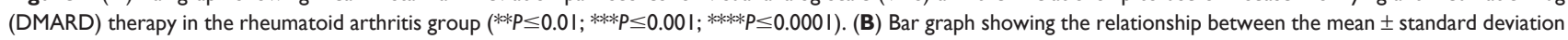
VAS and painDETECT questionnaire. Participants were grouped into three: unlikely neuropathic pain (UNP), possible neuropathic pain (PNP), and likely neuropathic pain (LNP) $(* * P \leq 0.01 ; * * * * P \leq 0.0001)$.

assess patients, including DAS28, blood tests, and clinical examinations. We asked participants to complete the questionnaire, which took an additional 10-15 minutes of their appointment, and patients reported that it was userfriendly and practical within a clinical setting. A further limitation is that we did not evaluate mood disorders in our study group. There is extensive literature (reviewed by Dougados et $\mathrm{al}^{35}$ ) to suggest that the emotional component of pain interferes with the efficacy of treatment. Future work is aimed at increasing the sample size, collecting data on mood disorders, and assessing test-retest reliability of the painDETECT questionnaire in RA in particular. 


\section{Conclusion}

In summary, the data from our study suggest that people with RA have higher pain sensitivity, with a smaller but significant number demonstrating pain sensitization at both joint and nonjoint sites using the painDETECT and VAS questionnaires. Our data concur with those of McWilliams et al, ${ }^{5}$ who reported high VAS scores in people with RA despite active treatment and DAS28 scores suggesting good control of disease activity. We suggest that the painDETECT score could be a useful tool in the clinic as a measure of the "sensitization" element to RA pain. Our findings suggest that implementation of multimodal pain assessment tools such as the VAS and painDETECT questionnaires in the arthritis clinic setting could open new avenues in the assessment and treatment of RA pain in the future.

\section{Acknowledgments}

The authors would like to thank Stefan Lazic and Mohsin Chaudhary for assistance with data collection. We would also like to thank the patients who gave their time to participate in this study. This work was funded by an Arthritis Research UK (ARUK) grant number 20163 and an Arthritis Research UK Medical Studentship to Tejal Magan. We acknowledge support from the National Institute of Health Clinical Research Network. We thank Professor Mike Hurley for useful discussions.

\section{Disclosure}

The authors report no conflicts of interest in this work.

\section{References}

1. Heiberg T, Kvein TK. Preferences for improved health examined in 1,024 patients with rheumatoid arthritis: pain has highest priority. Arthritis Care Res. 2002;47(4):391-397.

2. National Institute for Health and Care Excellence. Clinical guideline 79. Rheumatoid arthritis: the management of rheumatoid arthritis in adults [webpage on the Internet]; Feb 2009. Available from: nice.org.uk/cg79. Accessed August 27, 2014.

3. Aletaha D, Neogi T, Silman AJ, et al. 2010 rheumatoid arthritis classification criteria: an American College of Rheumatology/European League Against Rheumatism collaborative initiative. Ann Rheum Dis. 2010;69(9):1580-1588.

4. National Audit Office. Services for People With Rheumatoid Arthritis; 2009. Available from: http://www.nao.org.uk/wp-content/ uploads/2009/07/0809823.pdf. Accessed August 27, 2014.

5. McWilliams DF, Zhang W, Mansell JS, Kiely PD, Young A, Walsh DA. Predictors of change in bodily pain in early rheumatoid arthritis: an inception cohort study. Arthritis Care Res. 2014;64(10):1505-1513.

6. Borenstein D, Altman R, Bello A, et al. Report of the American College of Rheumatology Pain Management Task Force. Arthritis Care Res. 2010;62(5):590-599.

7. Kojima M, Kojima T, Suzuki S, et al. Depression, inflammation, and pain in patients with rheumatoid arthritis. Arthritis Rheum. 2009;61(8):1018-1024.

8. Merskey H. An Investigation of Pain in Psychological Illness [DM Thesis], Oxford; 1964.
9. Giordano J, Abramson K, Boswell M. Pain assessment: subjectivity, objectivity, and the use of neurotechnology. 1: Practical and ethical issues. Pain Physician. 2010;13:305-315.

10. Goldenberg DL, Clauw DJ, Fitzcharles M-A. New concepts in pain research and pain management of the rheumatic diseases. Semin Arthritis Rheum. 2011;41(3):319-334.

11. Katz WA, Rothenberg R. Treating the patient in pain. J Clin Rheumatol. 2005;11(2):S16-S28.

12. Freynhagen R, Baron R, Gockel U, Tölle TR. painDETECT: a new screening questionnaire to detect neuropathic components in patients with back pain. Curr Med Res Opin. 2006;22(10):1911-1920.

13. Gauffin J, Hankama T, Kautiainen H, Hannonen P, Haanpää M. Neuropathic pain and use of painDETECT in patients with fibromyalgia: a cohort study. BMC Neurol. 2013;13:21.

14. Morsø L, Kent PM, Albert HB. Are self-reported pain characteristics, classified using the painDETECT questionnaire, predictive of outcome in people with low back pain and associated leg pain? Clin J Pain. 2011;27(6):535-541.

15. Hochman JR, Davis AM, Elkayam J, Gagliese L, Hawker GA. Neuropathic pain symptoms on the modified painDETECT correlate with signs of central sensitization in knee osteoarthritis. Osteoarthritis Cartilage. 2013;21(9):1236-1242.

16. Fransen J, Creemers MCW, Van Riel PLCM. Remission in rheumatoid arthritis: agreement of the disease activity score (DAS28) with the ARA preliminary remission criteria. Rheumatology (Oxford). 2004;43(10): 1252-1255.

17. Hawker GA, Mian S, Kendzerska T, French M. Measures of adult pain: Visual Analog Scale for Pain (VAS Pain), Numeric Rating Scale for Pain (NRS Pain), McGill Pain Questionnaire (MPQ), Short-Form McGill Pain Questionnaire (SF-MPQ), Chronic Pain Grade Scale (CPGS), Short Form-36 Bodily Pain Scale (SF-36 BPS), and Measure of Intermittent and Constant Osteoarthritis Pain (ICOAP). Arthritis Care Res (Hoboken). 2011;63 Suppl 11:S240-S252.

18. Verkleij SPJ, Hoekstra T, Rozendaal RM, et al. Defining discriminative pain trajectories in hip osteoarthritis over a 2-year time period. Ann Rheum Dis. 2012;71(9):1517-1523.

19. Fransen J, van Riel PLCM. The Disease Activity Score and the EULAR response criteria. Rheum Dis Clin North Am. 2009;35(4):745-757, vii-viii.

20. Anderson JK, Anderson JK, Zimmerman L, Caplan L, Michaud K. Measures of rheumatoid arthritis disease activity: Patient (PtGA) and Provider (PrGA) Global Assessment of Disease Activity, Disease Activity Score (DAS) and Disease Activity Score with 28-Joint Counts (DAS28), Simplified Disease Activity Index (SDAI), Clinical Disease Activity Index (CDAI), Patient Activity Score (PAS) and Patient Activity Score-II (PASII), Routine Assessment of Patient Index Data (RAPID), Rheumatoid Arthritis Disease Activity Index (RADAI) and Rheumatoid Arthritis Disease Activity Index-5 (RADAI-5), Chronic Arthritis Systemic Index (CASI), Patient-Based Disease Activity Score With ESR (PDAS1) and Patient-Based Disease Activity Score without ESR (PDAS2), and Mean Overall Index for Rheumatoid Arthritis (MOI-RA). Arthritis Care Res (Hoboken). 2011;63 Suppl 11:S14-S36.

21. Porter D, Gadsby K, Thompson P, White J, McClinton C, Oliver S. DAS28 and rheumatoid arthritis: the need for standardization. Musculoskeletal Care. 2011;9(4):222-227.

22. Jawaheer D, Olsen J, Lahiff M. Gender, body mass index and rheumatoid arthritis disease activity: results from the QUEST-RA Study. Clin Exp Rheumatol. 2010;28(4):454-461.

23. Schaible H-G, Ebersberger A, Von Banchet GS. Mechanisms of pain in arthritis. Ann N Y Acad Sci. 2002;966:343-354.

24. Lee YC, Nassikas NJ, Clauw DJ. The role of the central nervous system in the generation and maintenance of chronic pain in rheumatoid arthritis, osteoarthritis and fibromyalgia. Arthritis Res Ther. 2011;13(2):211.

25. Pollard LC, Ibrahim F, Choy EH, Scott DL. Pain thresholds in rheumatoid arthritis: the effect of tender point counts and disease duration. $J$ Rheumatol. 2012:39(1):28-31. 
26. Woolf C. Central sensitization: implications for the diagnosis and treatment of pain. Pain. 2011;152:1-31.

27. Schweinhardt P, Kalk N, Wartolowska K, Chessell I, Wordsworth P, Tracey I. Investigation into the neural correlates of emotional augmentation of clinical pain. Neuroimage. 2008;40(2):759-766.

28. Rech J, Hess A, Finzel S, et al. Association of brain functional magnetic resonance activity with response to tumor necrosis factor inhibition in rheumatoid arthritis. Arthritis Rheum. 2013;65(2):325-333.

29. National Collaborating Centre for Chronic Conditions (UK). Rheumatoid arthritis: national clinical guideline for management and treatment in adults [webpage on the Internet]; 2009. London, UK: Royal College Of Physicians Of London. Available from: http://www.ncbi. nlm.nih.gov/pubmed/21413195. Accessed August 27, 2014.

30. Scott DL, Wolfe F, Huizinga TWJ. Rheumatoid arthritis. Lancet. 2010;376(9746):1094-1108.
31. Nurmohamed MT, Dijkmans BC. Are biologics more effective than classical disease-modifying antirheumatic drugs? Arthritis Res Ther. 2008;10(5):118

32. Quinn MA, Emery P. Window of opportunity in early rheumatoid arthritis: possibility of altering the disease process with early intervention. Clin Exp Rheumatol. 2003;21(5 Suppl 31):S154-S157.

33. Kidd BL, Langford RM, Wodehouse T. Arthritis and pain. Current approaches in the treatment of arthritic pain. Arthritis Res Ther. 2007;9(3):214.

34. Fitzcharles M-A,Almahrezi A, ShirY. Pain: understanding and challenges for the rheumatologist. Arthritis Rheum. 2005;52(12):3685-3692.

35. Dougados M, Soubrier M, Antunez A, et al. Prevalence of comorbidities in rheumatoid arthritis and evaluation of their monitoring: results of an international, cross-sectional study (COMORA). Ann Rheum Dis. 2014;73(1):62-68.
Journal of Pain Research

\section{Publish your work in this journal}

The Journal of Pain Research is an international, peer-reviewed, open access, online journal that welcomes laboratory and clinical findings in the fields of pain research and the prevention and management of pain. Original research, reviews, symposium reports, hypothesis formation and commentaries are all considered for publication.

\section{Dovepress}

The manuscript management system is completely online and includes a very quick and fair peer-review system, which is all easy to use. Visit http://www.dovepress.com/testimonials.php to read real quotes from published authors. 